\title{
Transcriptome profiling of the newborn mouse lung after hypoxia and reoxygenation: hyperoxic reoxygenation affects mTOR signaling pathway, DNA repair, and JNK-pathway regulation
}

Embjørg J. Wollen', Yngve Sejersted ${ }^{1,2}$, Marianne S. Wright ${ }^{1}$, Miroslaw Bik-Multanowski ${ }^{3}$, Anna Madetko-Talowska ${ }^{3}$, Clara-Cecilie Günther ${ }^{4}$, Ståle Nygård ${ }^{5}$, Przemko Kwinta ${ }^{6}$, Jacek J. Pietrzyk ${ }^{6}$ and Ola D. Saugstad ${ }^{1}$

BACKGROUND: The use of oxygen in acute treatment of asphyxiated term newborns is associated with increased mortality. It is unclear how hyperoxic reoxygenation after hypoxia affects transcriptional changes in the newborn lung.

METHODS: On postnatal day 7, C57BL/6 mice $(n=62)$ were randomized to 120-min hypoxia (fraction of inspired oxygen $\left(\mathrm{FiO}_{2}\right)$ 0.08) or normoxia. The hypoxia group was further randomized to reoxygenation for 30 min with $\mathrm{FiO}_{2}$ 0.21, 0.40, 0.60 , or 1.00 , and the normoxia group to $\mathrm{FiO}_{2} 0.21$ or 1.00 . Transcriptome profiling was performed on homogenized lung tissue using the Affymetrix 750k expression array, and validation was carried out by real-time polymerase chain reaction and enzyme-linked immunosorbent assay.

RESULTS: The hypoxia-reoxygenation model induced hypoxia-inducible factor 1 (HIF-1) targets like Vegfc, Adm, and Aqp 1. In total, $70 \%$ of the significantly differentially expressed genes were detected in the two high hyperoxic groups $\left(\mathrm{FiO}_{2}\right.$ 0.60 and 1.00). Reoxygenation with 100\% oxygen after hypoxia uniquely upregulated Gadd45g, Dusp1, Peg3, and Tgm2. Pathway analysis identified mammalian target of rapamycin (mTOR) signaling pathway, DNA repair, c-jun N-terminal kinase (JNK)-pathway regulation, and cell cycle after hyperoxic reoxygenation was applied.

CONCLUSION: Acute hypoxia induces HIF-1 targets independent of the reoxygenation regime applied. Hyperoxic reoxygenation affects pathways regulating cell growth and survival. DNA-damage-responsive genes are restricted to reoxygenation with 100\% oxygen.

S upraphysiological levels of $\mathrm{O}_{2}$ in neonates may induce proonged cellular dysfunctions with increasing morbidity $(1,2)$ and mortality (3). Hypoxia and reoxygenation generates higher levels of reactive oxygen species in the newborn lung when performed with supplemental oxygen (4), and DNA oxidation shows a dose-dependent increase in a model of hypoxia and graded fractions of inspired oxygen $\left(\mathrm{FiO}_{2}\right)(5)$. Hypoxic and hyperoxic stress even aggravate the development of lung injury in a long-term perspective (6). Recent evidence also shows that exposure to acute hyperoxia during only $30 \mathrm{~min}$ can increase mitochondrial oxidation in the fetal lung (7).

Preterm neonates are particularly susceptible to oxidative stress due to an immature intracellular antioxidant defense and presence of non-protein-bound iron, often in combination with ongoing infections (8). This can have long-term consequences contributing to the development of bronchopulmonary dysplasia (9). Current revised neonatal resuscitation guidelines recommend starting resuscitation with air for the term and near-term infant, but there is still no consensus on which $\mathrm{FiO}_{2}$ to administer to the preterm (10). Recent data (11) indicate lower oxidative stress and long-term lung injury after resuscitation of preterm born babies with 30 vs. $90 \%$ oxygen.

Gene expression changes after both brief (12) and prolonged hyperoxia (13) in the newborn lung. In the experimental bronchopulmonary dysplasia model, transcription of several genes involved in inflammation, cell cycle, and signal transduction is described (13). The mitogen-activated protein kinase signaling pathway may play an important role in hyperoxia-induced cell death (14), whereas the subfamily member c-jun $\mathrm{N}$-terminal kinase (JNK1/2) appears to be involved in reactive oxygen species -induced cell death (15). The mechanisms behind the detrimental effects of hypoxia-reoxygenation injury in the lungs are not completely understood, but experimental evidence indicates a worsening of hypoxic injury by reoxygenation with high $\mathrm{FiO}_{2}(12,16)$.

In this study, we have employed microarray analysis to assess transcriptional changes in lung tissue from newborn

'Department of Pediatric Research, Women and Children's Division, Oslo University Hospital, Rikshospitalet, University of Oslo, Oslo, Norway; ${ }^{2}$ Department of Microbiology, Division of Diagnostics and Intervention, Centre for Molecular Biology and Neuroscience, Oslo University Hospital, Oslo, Norway; ${ }^{3}$ Department of Medical Genetics, Polish-American Children's Hospital, Faculty of Medicine, Jagiellonian University, Krakow, Poland; ${ }^{4}$ Norwegian Computing Center, Oslo, Norway; ${ }^{5}$ Bioinformatics Core Facility, Institute for Medical Informatics, University of Oslo, Oslo University Hospital, Oslo, Norway; ${ }^{6}$ Department of Pediatrics, Polish-American Children's Hospital, Faculty of Medicine, Jagiellonian University, Krakow, Poland. Correspondence: Embjørg J. Wollen (ewollen@rr-research.no) 
mice in an early stage of alveolar development exposed to hypoxia followed by graded $\mathrm{FiO}_{2}$ supplementation during reoxygenation. We hypothesized that supplemental oxygen as compared with air induces differential gene expression changes and pathways.

\section{RESULTS}

\section{Validation of the Hypoxia Model}

Oxygen saturation, heart rate, pulse distention, and respiratory rate during hypoxia at 60 and $120 \mathrm{~min}$ are shown in Table 1. After $120 \mathrm{~min}$ of hypoxia, $\mathrm{pH}$ in the whole blood (mean $(\mathrm{SEM}))$ lowered to $7.23(0.04)(n=6)$ as compared with 7.42 (0.1) $(n=5)$ when exposed to air $(P<0.001)$. pH lowered with 0.13 from 60 to $120 \mathrm{~min}$ of hypoxia (17). Furthermore, base excess (mmol/l) lowered from $4.2(1.1)$ in air to $-13.3(1.7)$ $(P<0.001)$ after 120 -min hypoxia, and $\mathrm{pCO}_{2}$ lowered from $6.0(0.2)$ to $3.4(0.2)(P<0.001)$. Base excess lowered with 3.9 $\mathrm{mmol} / \mathrm{l}$ from 60 to $120 \mathrm{~min}$ of hypoxia (17).

\section{Overall Gene Expression Changes}

On postnatal day 7 (P7), 62 C57BL/6 mice from 10 litters, each comprising 6-10 pups, were included for gene expression analysis after excluding 9 , which died during hypoxia. The animals were randomized to either hypoxia or normoxia for $120 \mathrm{~min}$ followed by reoxygenation with different $\mathrm{FiO}_{2}$ (Figure 1). There were no significant weight (control $n=20 ; 3.9 \mathrm{~g}(0.5)$ vs.

Table 1. Physiological data in mice during exposure to $8 \%$ oxygen

\begin{tabular}{lccc}
\hline & $\begin{array}{c}\text { Air } \\
(n=6)\end{array}$ & $\begin{array}{c}\text { Hypoxia } \\
(n=6 ; 60 \mathrm{~min})\end{array}$ & $\begin{array}{c}\text { Hypoxia } \\
(n=4 ; 120 \mathrm{~min})\end{array}$ \\
\hline Oxygen saturation (\%) & $99 \pm 0.3$ & $49 \pm 2.8^{\text {tb }}$ & $42 \pm 3.3^{+}$ \\
Heart rate $(\mathrm{bpm})$ & $523 \pm 24$ & $660 \pm 20^{*}$ & $700 \pm 20^{* *}$ \\
Respiration rate $(\mathrm{brpm})$ & $196 \pm 20$ & $260 \pm 16^{*}$ & $216 \pm 18$ n.s. \\
Pulse distention $(\mu \mathrm{m})$ & $156 \pm 14$ & $118 \pm 17$ n.s. & $90 \pm 37$ n.s. \\
\hline
\end{tabular}

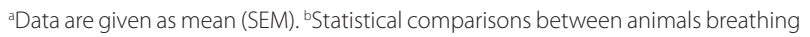
air and animals exposed to hypoxia for 60 or 120 min (two-tailed Student's $t$-test). ${ }^{*} P<$ $0.05 ;{ }^{* *} P<0.01{ }^{\dagger} P<0.001$

n.s., the $P$ values are not significant. hypoxia $n=42 ; 3.9 \mathrm{~g}(0.4)$ (mean (SEM)) or gender differences between the groups.

In the four groups following hypoxia (H21, H40, H60, and H100) as compared with controls (C21), 761 out of the 34,760 probes on the microarray chip were found to be significantly differentially expressed in whole-lung homogenates using the automatic thresholding rule by Bayesian ANOVA of microarrays (BAMarray) (18). Among the 24,674 probe sets with known gene symbol, 564 genes were significantly differentially expressed. Approximately $70 \%$ of the genes were altered in response to high $\mathrm{FiO}_{2}$ ( $\mathrm{H} 60$ and $\left.\mathrm{H} 100\right)$. The differentially expressed genes were distributed among the groups as follows: H21: 115, H40: 57, H60: 228, and H100: 164 (Figure 2a). The number was further reduced by setting the cutoff criteria at fold change $(\mathrm{FC}) \geq 1.2$ or $\leq 0.8$. The complete list of differentially expressed genes with cutoff after hypoxia and reoxygenation is presented in Supplementary Table S1 online. The expression patterns of genes that showed significant changes in at least one of the hypoxic groups as compared with controls (C21) were hierarchically clustered and can be viewed in Supplementary Figure S1 online.

As compared with reoxygenation with air (H21), the three groups receiving supplemental oxygen (H40, H60, and H100) had 430 of the 34,760 probes differentially expressed. Of these, 213 had known gene symbols; H40: 14 genes, H60: 141 genes, and H100: 58 genes. Further delimitation was performed as described above (Figure $\mathbf{2 b}$ and Supplementary Table S2 online).

Hypoxia induced almost twice as many genes with differential expression when succeeded by $100 \%$ oxygen (H100 vs. $\mathrm{C} 100 ; 228$ genes) than by air (H21 vs. C21; 115 genes). Further delimitation was performed as described above (Figure $2 \mathrm{c}$ and Supplementary Table S3 online).

In the group reoxygenated with $100 \%$ oxygen preceded by hypoxia (H100 vs. C21: 228 genes), nearly four times more genes with differential expression were induced than by hyperoxia per se (C100 vs. C21; 59). Further delimitation was performed as described above (Figure 2d and Supplementary Table S4 online).

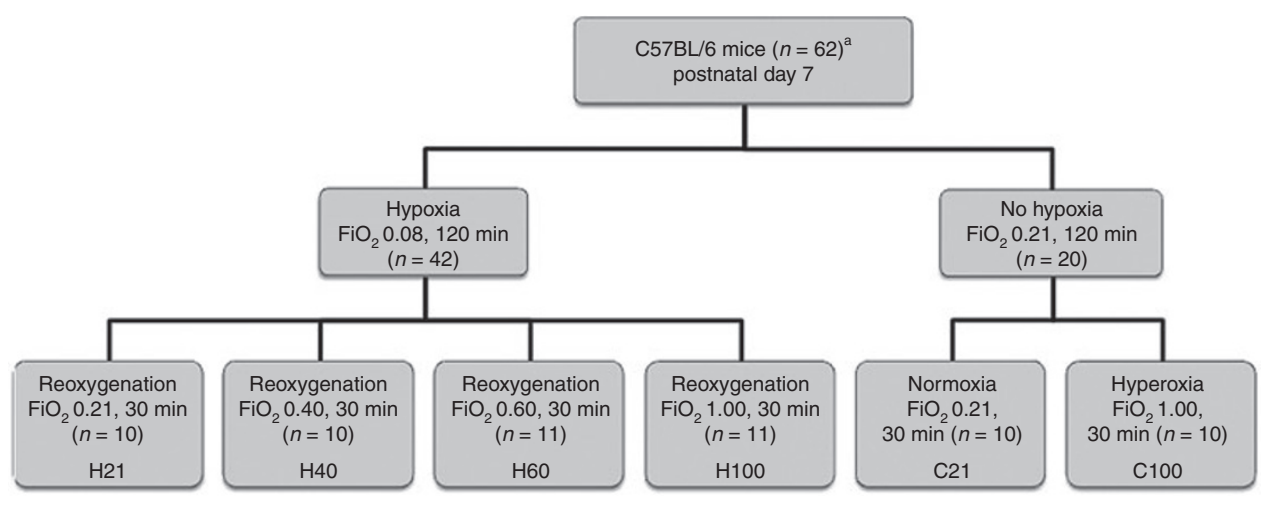

Figure 1. Experimental design of hypoxia-reoxygenation model. The following clinically relevant statistical comparisons were performed: (a) Groups $\mathrm{H} 21, \mathrm{H} 40, \mathrm{H60}$, and $\mathrm{H} 100$ were compared with controls (C21) to demonstrate the effect of hypoxia followed by graded FiO ${ }_{2}$ during reoxygenation. (b) Groups $\mathrm{H} 40, \mathrm{H} 60$, and $\mathrm{H} 100$ were compared with $\mathrm{H} 21$ to reveal the effect of supplemental oxygen during reoxygenation. (c) Group $\mathrm{H} 100$ was compared with C100 to show the effect of hypoxia when fraction of inspired oxygen 1.0 was administered. (d) Comparison between C100 vs. C21 was performed to depict the effect of hyperoxia per se. ${ }^{a}$ Out of 71 animals assigned to microarray, 9 animals died during hypoxia. 
a

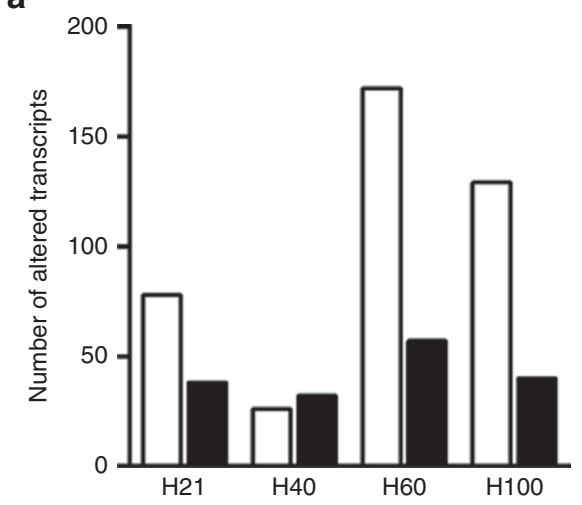

C

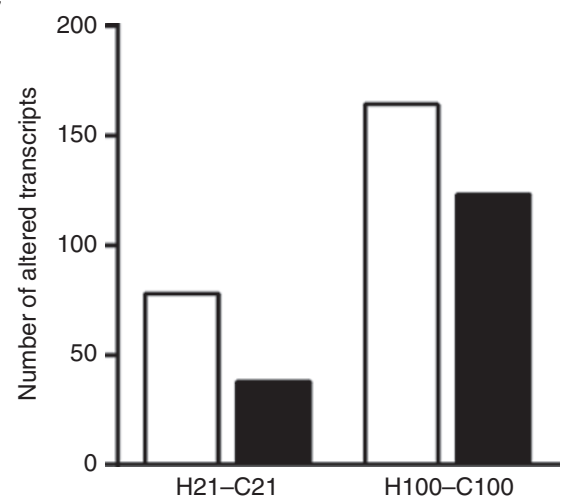

b

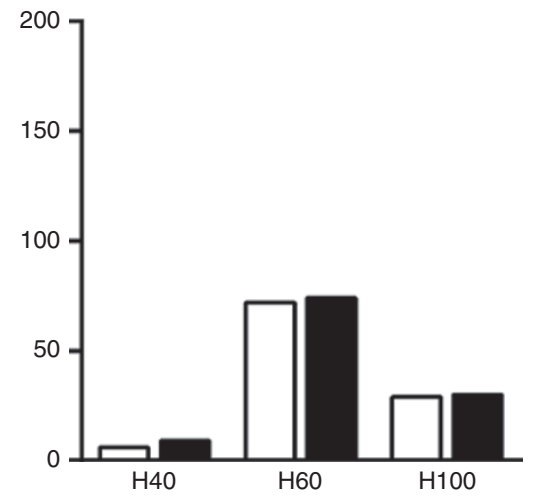

d

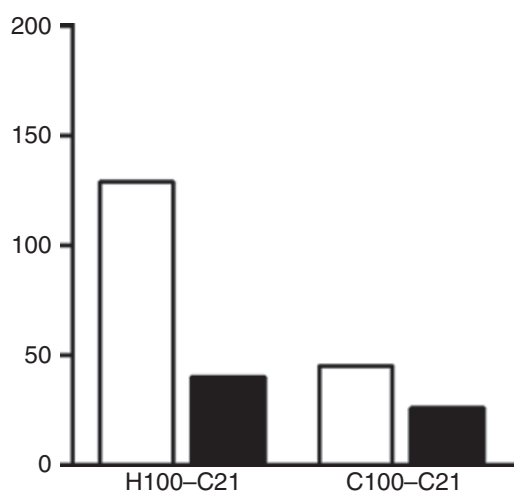

Figure 2. Overall gene expression. BAMarray analysis was used to determine (a) the total number of significantly altered genes identified as upregulated (white bars) or downregulated (black bars) in the four hypoxia groups compared with controls (C21), (b) the total number of significantly altered genes in mice reoxygenated with supplemental oxygen compared with the group reoxygenated with air $(\mathrm{H} 21)$, (c) the different effect of hypoxia when administered with air or fraction of inspired oxygen 1.0, and (d) the effect of hyperoxia with or without preceding hypoxia.

\section{Identification of Unique and Jointly Expressed Genes}

Comprehensive analysis of the four groups reoxygenated with different $\mathrm{FiO}_{2}$ preceded by hypoxia ( $\mathrm{H} 21, \mathrm{H} 40, \mathrm{H} 60$, and $\mathrm{H} 100$ ) and separately as compared with controls (C21) revealed both unique and overlapping results. Genes listed were selected based on their functions and FC $\geq 1.2$ (Figure 3a). Group H60 and H100 displayed 73 jointly expressed genes, of which 36 also were shared with group H21. The H40 group was distinctly different and shared only a few expressed genes with the three other hypoxia groups (data not shown).

When comparing $\mathrm{H} 21$ vs. $\mathrm{C} 21, \mathrm{H} 100$ vs. $\mathrm{C} 100$, and $\mathrm{C} 100$ vs. C21, many genes were expressed in both of the two first comparisons, whereas hyperoxia per se expressed other groups of genes (Figure $3 \mathbf{b}$ ).

\section{Pathways and Functional Categories}

Using gene set enrichment analysis (GSEA) with a cutoff false discovery rate $q$-value of $<0.10$, three Kyoto Encyclopedia of Genes and Genomes pathways and five Gene Ontology categories were found significantly altered in five different comparisons on a priori-selected gene sets (Table 2). The mammalian target of rapamycin (mTOR) signaling pathway was the only pathway upregulated in at least two comparisons, and 11 of 42 genes in the pathway were expressed in both the $\mathrm{H} 60$ and $\mathrm{H} 100$ comparison vs. C21 (Table 3). Upregulation in the two high
$\mathrm{FiO}_{2}$ groups dominated the pathway analysis, and genes contributing to the different pathways' significance are described in Supplementary Table S5 online.

Validation of Microarray Results With Real-Time Polymerase Chain Reaction

Seven transcripts were analyzed with real-time polymerase chain reaction (RT-PCR) in 40 of the same samples as used in the microarray study (Figure 4). The Pearson correlation coefficient was calculated for each of the seven transcripts selected for validation experiments, using FC relative to mean of C21 group for each individual. $r$ Values varied from 0.65 to 0.86 ( $P$ $<0.0001)$.

\section{Protein Validation}

Transcripts selected for protein validation were vascular endothelial growth factor $\mathrm{C}(\operatorname{Veg} f \mathcal{c})$, ataxia telangiectasia mutated homolog $(\mathrm{Atm})$, and neurotrophic tyrosine receptor kinase (Ntrk2) which also had been validated by RT-PCR. In addition, Lipocalin ( Lcn2) was selected independent of the RT-PCR analysis. VEGFC was significantly decreased in both the comparisons: $\mathrm{H} 21$ vs. $\mathrm{C} 21$ and $\mathrm{H} 100$ vs. C21. NTRK2 was significantly increased in the comparison of $\mathrm{H} 100$ vs. C21, but not in $\mathrm{H} 21$ vs. C21 (Figure 5). LCN2 and ATM did not show any significant differences in any of the comparisons. 
a
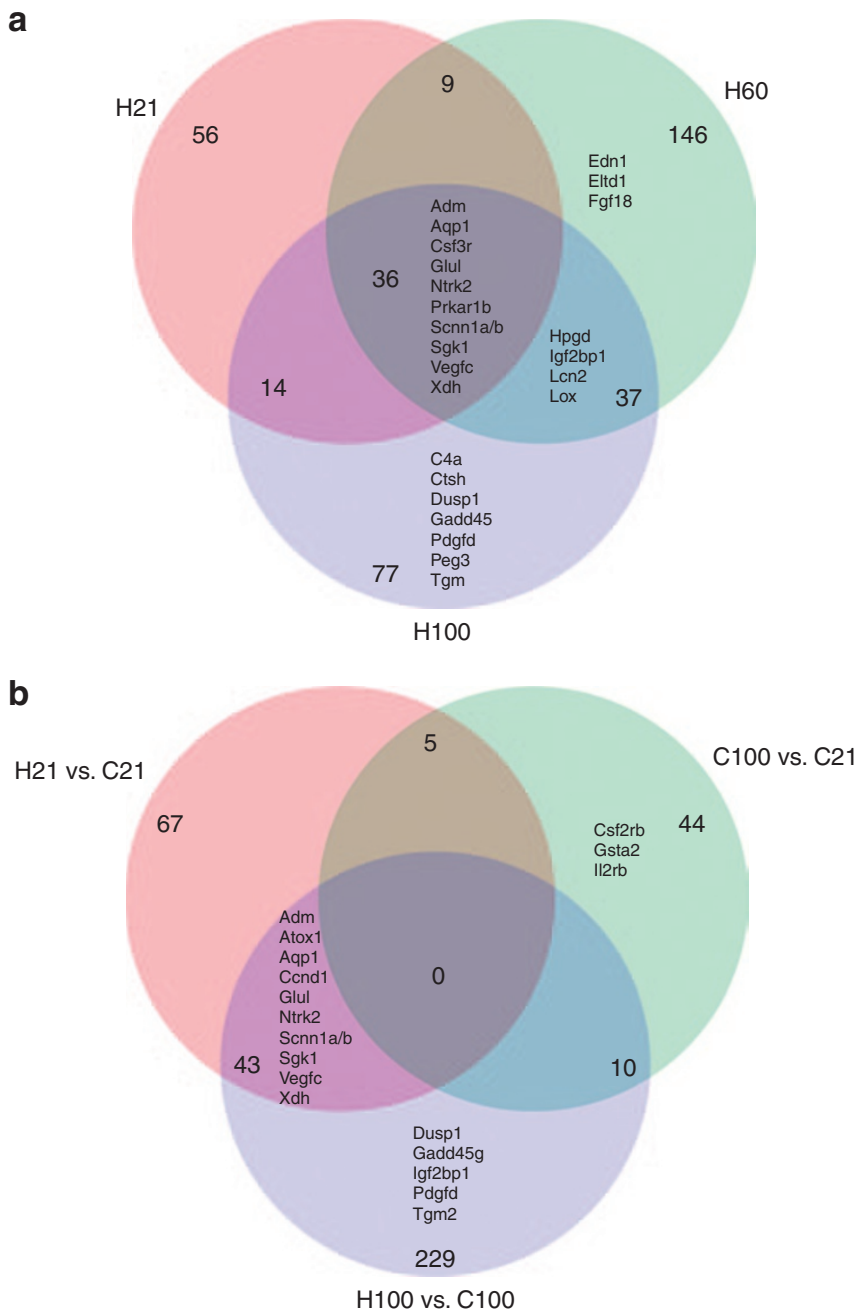

Figure 3. Unique and jointly expressed genes. Venn diagrams were used to show the number of differentially, jointly, and uniquely expressed genes based on the total number of significant genes. The genes listed in the figure are some of those with fold change $\geq 1.2$ and pivotal functions. (a) Comparisons of $\mathrm{H} 21, \mathrm{H} 60$, and $\mathrm{H} 100$ vs. $\mathrm{C} 21$ illustrating the unique number of genes for each condition in the outer circle, the jointly expressed genes are denoted in the intersections, and the genes in common for all three comparisons are represented in the centered intersection. The ones marked with an asterisk are discussed in more detail later in this article: Adm*, Aqp ${ }^{*}$, colony-stimulating factor 3 receptor (Csf3r), glutamine synthetase (Glul), Ntrk2*, protein kinase, cAMP-dependent regulatory, type I $\beta$ (Prkar1b), sodium channel, nonvoltage-gated $1 \alpha / \beta$ (Scnn1a/b), serum/ glucocorticoid-regulated kinase $1(\mathrm{Sgk} 1)$, Vegfc*, xanthine dehydrogenase (Xdh). Jointly expressed for $\mathrm{H} 60$ and $\mathrm{H} 100$ : hydroxyprostaglandin dehydrogenase 15 (Hpgd), Igf-2 mRNA-binding protein 1 (Igf2bp1), Lcn2*, Lox*. Unique for H60: endothelin 1 (Edn1), EGF, latrophilin seven transmembrane domain containing 1(Eltd1), fibroblast growth factor 18 (Fgf18). Unique for H100: complement component 4A (C4a), cathepsin (Ctsh), Dusp1* Gadd45g*, Pdgf, D polypeptide (Pdgfd), Peg3*, Tgm2*. (b) Results induced by $\mathrm{H} 21$ vs. $\mathrm{C} 21, \mathrm{C} 100$ vs. $\mathrm{C} 21$, and $\mathrm{H} 100$ vs. $\mathrm{C} 100$; antioxidant protein 1 (Atox 1), cyclin D1 (Ccnd1), colony-stimulating factor 2 receptor, $\beta$ (Csf2rb), glutathione $S$-transferase a 2 (Gsta2), II2 receptor, $\beta$ (Il2rb).

\section{DISCUSSION}

Perinatal asphyxia is a major cause of mortality, and survivors may suffer from multiorgan affection (19). Models of chronic hypoxia (18) or hyperoxia (13) demonstrate altered pulmonary morphology and gene expression patterns. In this study, our aim was to investigate the transcriptional changes from 120 min of hypoxia followed by reoxygenation with graded $\mathrm{FiO}_{2}$ supplemental oxygen, resembling resuscitation of an asphyxiated slightly preterm baby. We chose $30 \mathrm{~min}$ of reoxygenation because this is a typical resuscitation period used. The model inflicted significant metabolic acidosis after $120 \mathrm{~min}$ of hypoxia in line with the thresholds for defining intrapartum fetal asphyxia (20). The mice were in early alveolar stage of lung development, comparable to human lung tissue at $36 \mathrm{wk}$ of gestation (21).

Under these conditions, we found that the hypoxia-reoxygenation model used affected hypoxia-inducible factor 1 (HIF-1) targets. Hypoxia followed by reoxygenation with 60 or $100 \% \mathrm{O}_{2}$ induced an increased number of significantly differentially expressed genes as compared with those seen after low $\mathrm{O}_{2}(21$ and $40 \%)$. In total, $\sim 70 \%$ of the significantly differentially expressed genes were represented in the two high $\mathrm{FiO}_{2}$ reoxygenation regimes. Moreover, $100 \%$ oxygen preceded by hypoxia had a unique effect on DNA-damage-responsive genes. Pathway analysis revealed that hyperoxia after hypoxia induced significant pathways and functional categories involved in cell growth and survival; mTOR signaling pathway; DNA repair; regulation of the JNK signaling cascade; and cell cycling. Microarray results were confirmed by RT-PCR showing strong positive statistical correlation, indicating that the method is trustable in measuring gene expression in this model of hypoxia and reoxygenation.

The difference in the expression measured as a FC in the majority of genes ranged between 1.1 and 1.5, which probably reflects the short duration of hypoxia and hyperoxia used herein. We chose a FC limit of $1.2 \geq$ and $\leq 0.8$ since it has been shown that even a $20 \%$ difference in gene expression can be clinically significant and sufficient to actually induce changes in protein (22). Studies using microarray technique with the approximate stimuli on rodents have also limited the FC to $1.2(23)$.

In the GSEA analysis, genes were included that displayed subtle alterations in expression levels based on the knowledge that these genes might be of importance due to their biological function and involvement in pathways. The $q$ value is the value obtained by adjusting the original $P$ values for multiple testing by controlling the false discovery rate.

\section{Hypoxia-Induced Transcriptional Changes}

Adaption to low oxygen levels is primarily regulated at the transcriptional level by HIF-1 and induces the expression of several up- and downstream targets. HIF-1-responsive genes jointly expressed in the groups reoxygenated with air; 60 or $100 \% \mathrm{O}_{2}$ were upregulated in the current hypoxia-reoxygenation model. Vegfc is a HIF-1 target playing an important role in the pathogenesis of pulmonary inflammation in the preterm infant (24). Our findings are in partial agreement with those by Remesal et al. (25), who showed that Vegf expression increases in lung tissue from newborn rats exposed to acute hypoxia $\left(10 \% \mathrm{O}_{2} ; 120 \mathrm{~min}\right)$ and decreases when hypoxia is followed by hyperoxia (>95\%; $120 \mathrm{~min}$ ). We found an increase of $\mathrm{Vegfc}$ 


\section{Articles | Wollen et al.}

Table 2. Pathway analysis with false discovery rate (FDR) $q$ value $<0.10$

\begin{tabular}{|c|c|c|c|c|c|}
\hline Database & Pathways & Statistical comparison ${ }^{\mathrm{a}}$ & Type of regulation & FDR $q$ value $^{\mathrm{b}}$ & NESc \\
\hline \multirow[t]{5}{*}{ KEGG } & \multirow[t]{2}{*}{ DNA polymerase } & $\mathrm{H} 21$ vs. $\mathrm{C} 21$ & Down & 0.006 & -1.85 \\
\hline & & H40 vs. C21 & Down & 0.011 & -1.82 \\
\hline & \multirow[t]{2}{*}{ Mammalian target of rapamycin signaling pathway } & H60 vs. C21 & Up & 0.033 & 1.86 \\
\hline & & H100 vs.C21 & Up & 0.081 & 1.79 \\
\hline & Double strand break repair & H40 vs. C21 & Down & 0.033 & -1.82 \\
\hline \multirow[t]{4}{*}{ Gene ontology } & Regulation of DNA replication & H40 vs. C21 & Down & 0.038 & -1.70 \\
\hline & DNA repair & H40 vs. C21 & Down & 0.052 & -1.72 \\
\hline & Positive regulation of c-jun $\mathrm{N}$-terminal kinase activity & H60 vs. C21 & Up & 0.008 & 2.01 \\
\hline & Nucleotide excision repair & H60 vs. C21 & Up & 0.067 & 1.77 \\
\hline
\end{tabular}

aStatistical comparison of group pairs. ${ }^{\circ}$ False discovery rate $q$ value. ${ }^{` N}$ ormalized enrichment score.

KEGG, Kyoto Encyclopedia of Genes and Genomes.

Table 3. Mammalian target of rapamycin signaling pathway: the 11 transcripts shared by the groups reoxygenated with fraction of inspired oxygen 0.60 and 1.00

\begin{tabular}{|c|c|c|c|c|}
\hline Gene name & Gene symbol & Function & $\mathrm{S} 2 \mathrm{~N}^{\mathrm{a}} \mathrm{H} 60 / \mathrm{H} 100$ & RES $^{b} \mathrm{H} 60 / \mathrm{H} 100$ \\
\hline Vascular endothelial growth factor C & Vegfc & Growth factor & $0.148 / 0.172$ & $0.123 / 0.156$ \\
\hline Placental growth factor & Pgf & Growth factor & $0.129 / 0.162$ & $0.129 / 0.306$ \\
\hline Eukaryotic translation initiation factor 4 E binding protein 1 & Eif4ebp1 & Regulation of protein translation & $0.021 / 0.052$ & $0.426 / 0.313$ \\
\hline DNA-damage-inducible transcript 4 & Ddit4 & Inhibition of cell growth & $0.028 / 0.040$ & $0.375 / 0.298$ \\
\hline Phosphoinositide-3-kinase, catalytic, $\Delta$ polypeptide & Pik3cd & Signal transduction & $0.042 / 0.037$ & $0.282 / 0.348$ \\
\hline Unc-51-like kinase 3 & Ulk3 & Signal transduction & $0.022 / 0.032$ & $0.391 / 0.347$ \\
\hline Protein kinase, AMP-activated, a 1 catalytic subunit & Prkaa1 & Catalytic processes & $0.058 / 0.031$ & $0.219 / 0.368$ \\
\hline Unc-51-like kinase 2 & Ulk2 & Catalytic processes & $0.040 / 0.030$ & $0.341 / 0.391$ \\
\hline Phosphoinositide-3-kinase, regulatory subunit 3 & Pik3r3 & Signal transduction & $0.041 / 0.029$ & $0.309 / 0.406$ \\
\hline
\end{tabular}

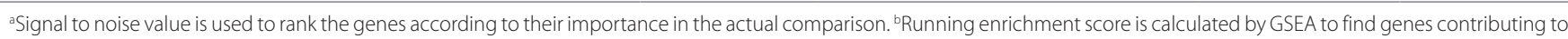
pathways significance.

GSEA, gene set enrichment analysis.

when hypoxia was followed by hyperoxia $\left(60\right.$ and $\left.100 \% \mathrm{O}_{2}\right)$. This discrepancy may be due to a shorter exposure time to hyperoxia $(30 \mathrm{~min})$. The protein level of VEGFC decreased significantly after reoxygenation with air and was close to significant when $100 \% \mathrm{O}_{2}$ was used $(P=0.06)$. We speculate that stored VEGFC is rapidly depleted. Replenishing VEGF protein has been shown to be dependent on nuclear factor- $\mathrm{\kappa B}$ activation and translocation into the nucleus. This procedure may take $6 \mathrm{~h}$ to $3 \mathrm{~d}$, depending on the severity of hypoxia/hyperoxia (26).

Other HIF-1 target genes, such as adrenomedullin $(A d m)$, Ntrk2, and aquaporin 1 (Aqp1), displayed increased expression after hypoxia followed by reoxygenation with air, 60 or $100 \% \mathrm{O}_{2}$. The $\mathrm{Adm}$ gene product is a multifunctional regulatory peptide implicated among others in the cellular response to inflammation and induction of angiogenesis. In accordance with our results, $A d m$ expression is upregulated by hypoxia in lung tissue of rats both in vitro and in vivo (27). Ntrk2, encoding the receptor for brain-derived neurotrophic factor, has also been shown to be elevated at both mRNA and protein levels in hypoxic $\left(8 \% \mathrm{O}_{2} ; 6 \mathrm{~h}\right)$ vs. normoxic rat lungs $(28)$. This is in line with our findings showing an increase of NTRK2 when $100 \%$ $\mathrm{O}_{2}$ is used during reoxygenation after hypoxia. Furthermore, the water channel AQP1 involved in gas permeation is highly expressed in tissues with rapid $\mathrm{O}_{2}$ transport and is reported to be upregulated at both mRNA and protein levels after hypoxia in lung tissue of mice (29).

\section{Hyperoxia-Induced Transcriptional Changes}

Hyperoxic reoxygenation with 60 or $100 \% \mathrm{O}_{2}$ induced changes in Lcn2 and Lysyl oxidase (Lox) transcript levels. The acutephase glycoprotein LCN2 is active under inflammation, apoptosis, and suppression of bacterial growth. There is recent evidence that $L c n 2$ also has a pivotal role in the protection against oxidative stress during hypoxia/reoxygenation in vitro (30) and that it acts as an antioxidant (31). Still, we could not detect 
a

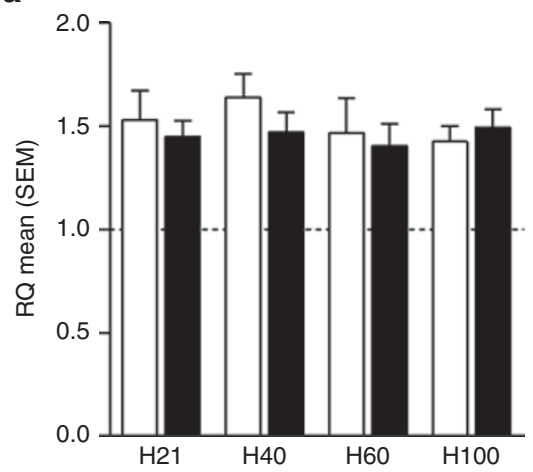

d

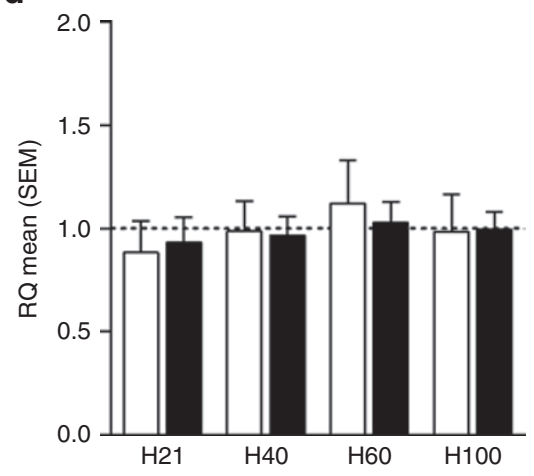

b

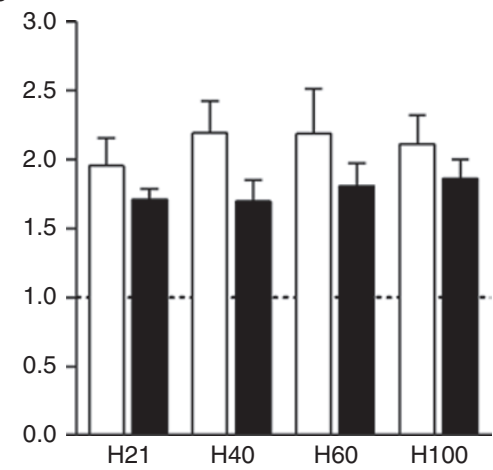

e

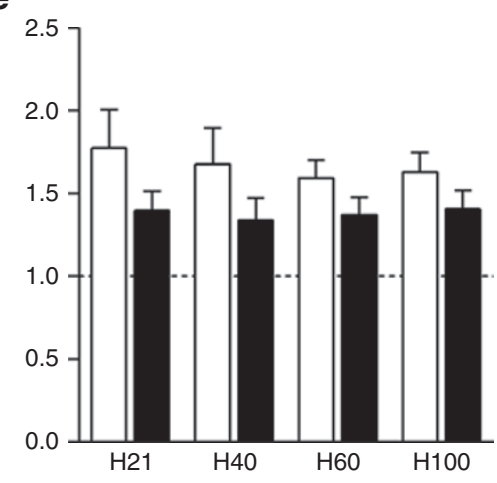

C

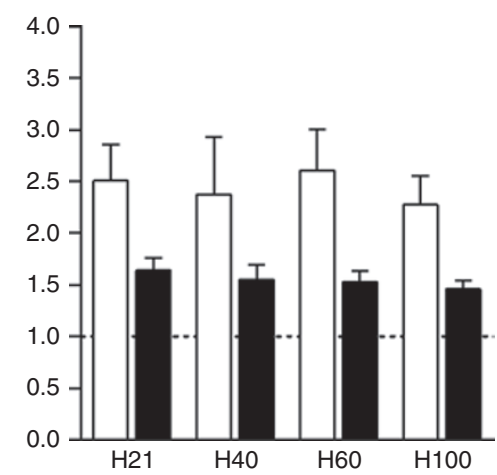

f

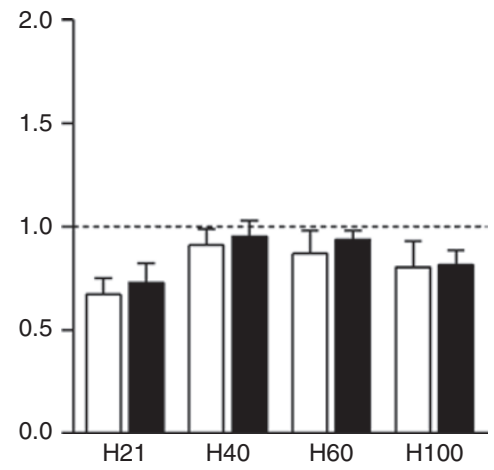

g

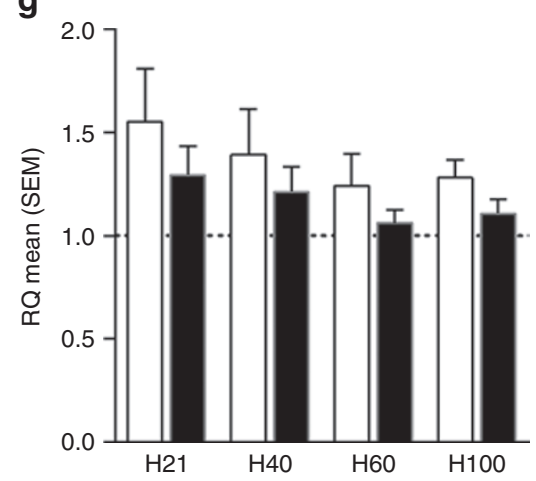

Figure 4. Validation of microarray results by real-time polymerase chain reaction. (a-g) Gene expression after hypoxia followed by graded fraction of inspired oxygen as compared with controls for seven selected genes. Relative gene expression signal levels determined by microarray (black bars) as compared with levels determined by real-time polymerase chain reaction (white bars). These data are expressed as mean fold change for the hypoxia groups as compared with controls (C21, dotted line). The error bars are SEM. Pearson correlation coefficient is calculated for each gene. (a) Vegfc $(r=0.66$; $P<0.0001)$, (b) Adm $(r=0.84 ; P<0.0001)$, (c) Ntrk2 $(r=0.73 ; P<0.0001)$, (d) Atm $(r=0.86 ; P<0.0001)$, (e) Pgf $(r=0.71 ; P<0.0001)$, (f) Chek1 $(r=0.65 ; P<$ $0.0001)$, and (g) Ddit4 ( $r=0.82 ; P<0.0001)$.

increased levels of LCN2 after hypoxia and reoxygenation. Lox, a copper oxidase, showed increased expression and activity after excessive hyperoxia exposure in an animal model of bronchopulmonary dysplasia and in humans with the same condition (32). We found an upregulation of Lox expression, indicating early activation which may affect long-term outcome.

Reactive oxygen species may oxidize macromolecules including DNA and RNA. Transcripts induced only by $100 \%$ $\mathrm{O}_{2}$ during reoxygenation were predominantly involved in the oxidative stress-induced DNA-damage response, including growth arrest and DNA-damage-inducible $45 \gamma$ (Gadd45g), dual specificity phosphatase 1 (Dusp1), paternally expressed
3 (Peg3), and transglutaminase $2(\operatorname{Tgm} 2)$. In agreement with our findings, hyperoxia increases Gadd45g expression in adult mouse lungs (26). Taken together, our results are indicative of an early DNA-damage response in the lungs after oxidative stress.

Hypoxia and DNA damage are both stress signals that can initiate cellular responses to exert various functions like mTOR pathway, apoptosis, DNA repair, cell cycle arrest, and antioxidant defence (33). The mTOR signaling pathway plays a crucial role in the regulation of cell proliferation, survival, and energy metabolism in response to stress. Herein, mTOR signaling pathway was the only pathway upregulated in response 
a

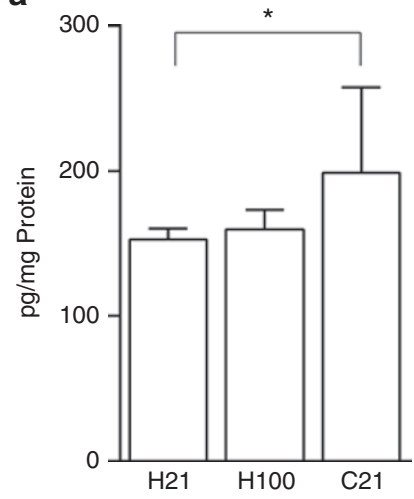

b

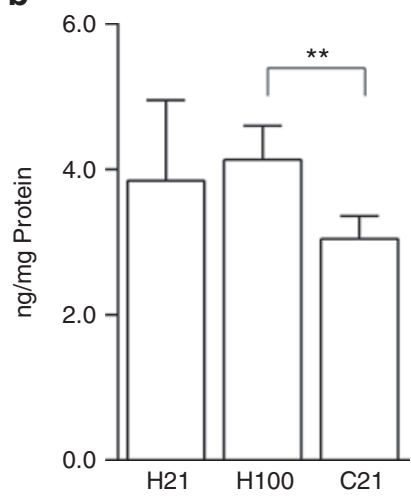

Figure 5. Protein analysis. Protein levels after hypoxia followed by reoxygenation with air or $100 \% \mathrm{O}_{2}$ as compared with controls. (a) VEGFC is significantly decreased in $\mathrm{H} 21$ as compared with controls $(P=0.04)$, whereas $\mathrm{H} 100$ vs. $\mathrm{C} 21$ increased $(P=0.06)$. (b) NTRK2 is significantly increased in $\mathrm{H} 100$ as compared with controls $(P=0.001)$, whereas $\mathrm{H} 21$ vs. C21 decreased $(P=0.15)$. Data are given as mean values, and the error bars are SEM. ${ }^{*} P<0.05 ;{ }^{* *} P<0.01$.

to both 60 and $100 \% \mathrm{O}_{2}$. These treatments mimic the increased oxidative stress the lung tissue of the newborns is exposed to in a resuscitation situation. Because both hypoxia through activation of p53 (33) and hyperoxia (34) can affect the mTOR signaling pathway, the genes involved herein are predominantly growth factors and signal transducers which could trigger beneficial responses to promote cell survival and diminish toxicity.

Hyperoxic reoxygenation also increases the activity of JNK (35), responding to stress stimuli such as inflammation and hyperoxia. An upregulation of the functional category "positive regulation of JNK" after $60 \% \mathrm{O}_{2}$ indicates that a stressful hypoxic event followed by hyperoxia in the newborn mouse lung tissue activates JNK signaling pathway. This activation can lead to reactive oxygen species -induced cell death (13).

Both DNA repair and replication are influenced by hypoxia and reoxygenation. In the current study, DNA replication was downregulated after 21 and $40 \% \mathrm{O}_{2}$ reoxygenation, which may indicate damage to the DNA. An additional indication of DNA-damage response was upregulation of nucleotide excision repair after hyperoxic reoxygenation with $60 \% \mathrm{O}_{2}$. This DNA repair pathway removes bulky DNA lesions that may cause cell cycle arrest or genomic instability (36). With the exception of lung tissue, indicating tissue-specific nucleotide excision repair adaptability, bulky lesions accumulate in rats during the first hours after normal birth (37). Hence, our findings may be epiphenomenal to an increased amount of DNA damage. Our group has previously reported that increasing $\mathrm{FiO}_{2}$ during resuscitation of newborn pigs results in increasing levels of 8-oxodG in urine, which is a marker of oxidative stress (5). Moreover, there is a positive correlation between reoxygenation time and 8-oxoguanine in mouse liver DNA after hypoxia (17).

DNA damage and repair influence the cell cycle pathway depending on the type of damage and repair mechanisms needed. The cell cycle pathway showed here the upregulation in the comparison between the two groups reoxygenated

with $60 \% \mathrm{O}_{2}$ and air. Hyperoxia is known to modulate genes involved in cell cycle regulation, and cell cycle arrest is the most commonly observed phenomenon during hyperoxia (38). We observed indeed an activation of the important checkpoints responsible for cell cycle arrest: Chek1, Chek2, and Bub1.

Studies on long-term (days) exposure to hyperoxia report more drastic effects than those reported herein. However, in this explorative study, only one observation point was deliberately examined, precluding the ability to reveal long-term consequences of hypoxia and reoxygenation in the lung. Protein analysis at the same time point as the mRNA measurements also limit the long-term study of acute mRNA changes. A follow-up study is being conducted, studying a temporal profile of genes at mRNA and protein level.

\section{Conclusion}

This study provides a global overview of gene expression in the newborn mouse lung in the early alveolar stage following acute hypoxia and graded $\mathrm{FiO}_{2}$ during reoxygenation. Acute hypoxia induces HIF-1 targets independent of the following reoxygenation regime. Hyperoxic reoxygenation mediates, in total, $\sim 70 \%$ of the gene expression changes observed, and GSEA analysis also indicates further activation of the mTOR signaling pathway, DNA repair, JNK-pathway regulation, and cell cycle. Reoxygenation with 100\% oxygen preceded by hypoxia affects several genes involved in oxidative stressinduced DNA-damage processing.

\section{METHODS}

\section{Animals}

All experiments were approved by the Norwegian Animal Research Authority. The animals were cared for and handled in accordance with European Guidelines for Use of Experimental Animals by Federation of European Laboratory Animals Science Association. C57BL/6 mice were received from the Taconic Facility in Tornbjerg, Denmark. Mice were stabled and bred at $24^{\circ} \mathrm{C}$ on a 12:12 light/dark cycle with access to a diet of pellets and water ad libitum.

\section{Validation of the Model}

To validate the hypoxia model, the two following separate experiments were performed: (i) physiological parameters: heart rate, respiratory rate, $\mathrm{SpO}_{2}$, and pulse distention were monitored in $\mathrm{P} 7(n=6)$ during hypoxia for 120 min using Mouse Ox (GeneChip Sciences, Oakmont, PA). (ii) Whole blood was collected from P7 $(n=17)$, randomized to hypoxia (as described under the next section) for $120 \mathrm{~min}$ or to normoxia (control). Mice were decapitated immediately after hypoxia, and blood collected from the severed neck vessels was analyzed for $\mathrm{pH}$, base excess, and $\mathrm{pCO}_{2}$ (Model ABL-800; Radiometer America, Westlake, $\mathrm{OH}$ ).

\section{Hypoxia and Reoxygenation}

Custom-made lucite chambers were used for hypoxia and reoxygenation experiments. Hypoxia was performed with premixed gas: $8 \%$ oxygen balanced with nitrogen (Yara, Norway). Following hypoxia, reoxygenation with different $\mathrm{FiO}_{2}$ levels was performed simultaneously in separate chambers. During reoxygenation, $\mathrm{FiO}_{2}$ was monitored with conventional oxygen mixers. Atmospheric pressure was maintained constant by a continuous supply of $\mathrm{O}_{2}$ at $0.5 \mathrm{l} / \mathrm{min}$ and open outlets. $\mathrm{CO}_{2}$ concentrations were $<0.3 \%$. The temperature in the chambers was kept at $35 \pm 0.5^{\circ} \mathrm{C}$ by floor heating and humidity maintained at $40-50 \%$ with an open water source. Pups were separated from their dams throughout the experiment. The mice were not anesthetized or restrained. 
Sixty-two C57BL/6 mice P7, from 10 different litters, were randomized to hypoxia $\left(\mathrm{FiO}_{2} 0.08\right)$ or normoxia $\left(\mathrm{FiO}_{2} 0.21\right)$ for $120 \mathrm{~min}$. The hypoxia group was further randomized to subsequent reoxygenation for $30 \mathrm{~min}$ with $\mathrm{FiO}_{2} 0.21(\mathrm{H} 21), 0.40(\mathrm{H} 40), 0.60(\mathrm{H} 60)$, or 1.00 (H100), and the normoxia group to either $\mathrm{FiO}_{2} 0.21$ (C21) or 1.00 (C100) ( $n=10-11$ in each group). Following $150 \mathrm{~min}$ in air, decapitation and rapid dissection on ice were performed. Whole-lung tissue was subsequently preserved in RNAlater stabilization reagent at $-20^{\circ} \mathrm{C}$ (Qiagen, Valencia, CA).

\section{Tissue Preparation}

Samples were thawed at room temperature and homogenized in 350- $\mu$ l RTL buffer (Qiagen). Total RNA was then extracted using an RNeasy Micro Kit (Qiagen). RNA concentration was measured by a NanoDrop spectrophotometer ND-1000 (Thermos Fisher Scientific, Wilmington, DE), and RNA quality was determined by a 2100 Bioanalyzer (Agilent, Santa Clara, CA).

\section{Microarray Analysis}

Hundred nanograms of total RNA was used from each animal $(n=$ 62). Mouse Gene 1.0 ST Arrays (Affymetrix, Santa Clara, CA) comprise more than 750,000 unique 25-mer oligonucleotide features constituting more than 28,000 gene-level probe sets. GeneChip Whole Transcript Sense Target Labeling Assay Manual, version 4, was used (Affymetrix). This Affymetrix Assay generates amplified and biotinylated sense-strand DNA targets from the entire expressed genome. After hybridization, arrays were registered in GeneChip Operating Software, washed, and stained with the use of GeneChip Fluidics Station 450 and FS450_0007 protocol. Arrays were then scanned with the GeneChip Scanner 3000 7G (Affymetrix) controlled by GeneChip Operating Software. For further statistical analysis, Gene Chip Operating Software was used (Supplementary Material, section 2 online).

\section{Primer Design and RT-PCR}

RT-PCR was performed in 40 original samples (H21, $n=7$; H40, $n=5$; $\mathrm{H} 60, n=9$; H100; $n=10$; and C21, $n=9$ ), which had sufficient cDNA for the analysis, to verify differentially expressed transcripts detected by microarray. The mouse DNA sequences for the seven transcripts (chosen based on BAMarray and GSEA analysis) selected for validation: Vegfc, Adm, Ntrk2, Atm, Pgf, Chek1, and Ddit4, were known at the time of the present study. Primers were designed using Primer Express 3.0 Software (Applied Biosystems, Carlsbad, CA) (Supplementary Table S6 online). Total mRNA was extracted, reverse transcribed, and amplified as described previously (39). RT-PCR was performed with 50-ng cDNA and 400-nmol/l primers for the target genes and reference gene PPIA (peptidylprolyl isomerase A) employing the SYBR Green PCR Master mix in an ABI PRISM 7300 Real-time PCR System, universal instrument setting (Applied Biosystems) Data were analyzed by the comparative $\mathrm{Ct}$ method of relative quantification $\left(2^{-\Delta \Delta C t}\right)(40)$.

\section{Protein Analysis}

A separate experiment was performed for protein analysis. P7 mice ( $n$ $=21)$ were randomized to hypoxia $(n=16)$ or normoxia $(n=5, \mathrm{C} 21)$. The hypoxia group was further randomized to reoxygenation with either $\mathrm{FiO}_{2} 0.21(\mathrm{H} 21)$ or 1.00 (H100). The experiment followed the same experimental design as previously described and lungs were harvested after $150 \mathrm{~min}$. Frozen lung tissue was homogenized in lysis buffer (Tris-HCL) containing 1\% NP-40 and a protease inhibitor cocktail (Roche Diagnostics, Germany), using omnitip (OMNI International, Kennesaw, GA). VEGFC (Mouse VEGFC ELISA kit Biorbyt, UK), ATM (ATM ELISA kit, USCN Life Science, Wuhan, China), NTRK2 (NTRK2 (Mouse) ELISA kit, Abnova, Taiwan), and LCN2 (NGAL Mouse Duo Set ELISA, R\&D Systems, Minneapolis, MN) were used.

\section{Statistical Analysis}

For validation of the experiments of the hypoxiamodel and the protein analysis, a $t$-test were used for comparison between groups. $P<$ 0.05 was considered statistically significant. Pearson correlation analysis was performed to examine the correlation between the RT-PCR and the microarray results, using FC relative to mean of $\mathrm{C} 21$ group for each individual.
$\mathrm{R} /$ Bioconductor package robust multiarray average (Fred Hutchinson Cancer Research Center, Seattle, WA) was used to preprocess the microarray data (41). In robust multiarray average, raw intensity values are background corrected, $\log 2$ transformed, and then quantile normalized, before an additive linear model is applied to the normalized data. The result is an expression measure for each probe set on each array. Arrays were preprocessed together to ensure comparability across conditions.

The BAMarray method was used to identify differentially expressed genes (42). This method uses a Bayesian test statistic and balances the number of falsely detected genes and falsely nondetected genes, i.e., it controls both the false discovery rate and the false nondiscovery rate. The cutoff value that results in an optimal balance is determined automatically from the data, contrary to most other software packages that require the users to specify a cutoff value for the test statistic. In BAMarray, the expression values for all probe sets are taken into account and result in a list of differentially expressed probe sets or genes as indicated by their known gene symbol. However, some genes are represented by several probe sets but have the same gene symbol. To determine the number of regulated genes, we counted the number of genes for which at least one probe set was up- or downregulated. To reduce the number of genes to be further discussed, cutoff criteria were set at $\mathrm{FC} \geq 1.2$ or $\leq 0.8$.

In the cluster analysis, genes were clustered according to similarity in expression values. Average FC for genes being significantly expressed in at least one of the four hypoxia groups vs. controls (C21) were clustered using Pearson correlation as distance measure.

GSEA is a method to determine whether a set of a priori defined gene sets is significantly enriched in one group as compared with another (43). The GSEA analysis was performed on the preprocessed and normalized data for the probe sets with known gene symbols only. GSEA uses data from all genes in a gene set in a weighted fashion to determine whether the gene set is significantly regulated. Therefore, the analysis was performed independent of the BAMarray analysis, which provides a dichotomous answer to whether each gene is significantly regulated. The median of the probe set intensities was used for probes sharing gene symbols. The gene sets were selected among the Kyoto Encyclopedia of Genes and Genomes pathway database (Kanehisa Laboratories, Japan) and Gene Ontology gene sets available in the Molecular Signatures Database (MSigDB v2.5; GO, Stanford, CA). A weighted Kolmogorov-Smirnovlike statistic was calculated for each gene set, and statistical significance was assessed by permutation tests. To adjust for multiple hypotheses testing when several gene sets are considered, the false discovery rate is controlled. All primary microarray data are MIAME (Minimum Information About A Microarray Experiment) compliant and submitted to the Gene Expression Omnibus (44) (accession number GSE32500).

\section{SUPPLEMENTARY MATERIAL}

Supplementary material is linked to the online version of the paper at http:// www.nature.com/pr

\section{ACKNOWLEDGMENTS}

We express gratitude to Monica Atneosen-Aasegg and Grethe Dyrhaug for their assistance during the animal experiments and RT-PCR analysis. We also thank Grethe Dyrhaug and Maren Bakkebø for their contribution with the protein analysis. The animal experiments were performed at the Centre of Comparative Medicine, Oslo University Hospital, Rikshospitalet, and we appreciate the help and facilities that we were offered during our work.

\section{STATEMENT OF FINANCIAL SUPPORT}

Financial Mechanism of European Economic Area (PL0226), Polish Ministry of Science (E023/P01/2008/02/85), and South-Eastern Regional Health Authority, Norway.

Disclosure: The authors declared no conflict of interest.

\section{REFERENCES}

1. Yee M, White RJ, Awad HA, Bates WA, McGrath-Morrow SA, O’Reilly MA. Neonatal hyperoxia causes pulmonary vascular disease and shortens life span in aging mice. Am J Pathol 2011;178:2601-10.

2. Saugstad OD. Is oxygen more toxic than currently believed? Pediatrics 2001;108:1203-5. 
3. Saugstad OD, Ramji S, Soll RF, Vento M. Resuscitation of newborn infants with $21 \%$ or $100 \%$ oxygen: an updated systematic review and meta-analysis. Neonatology 2008;94:176-82.

4. Kondo M, Itoh S, Isobe K, et al. Chemiluminescence because of the production of reactive oxygen species in the lungs of newborn piglets during resuscitation periods after asphyxiation load. Pediatr Res 2000;47(4 Pt 1):524-7.

5. Solberg R, Andresen JH, Escrig R, Vento M, Saugstad OD. Resuscitation of hypoxic newborn piglets with oxygen induces a dose-dependent increase in markers of oxidation. Pediatr Res 2007;62:559-63.

6. Ratner V, Slinko S, Utkina-Sosunova I, Starkov A, Polin RA, Ten VS. Hypoxic stress exacerbates hyperoxia-induced lung injury in a neonatal mouse model of bronchopulmonary dysplasia. Neonatology 2009;95:299305.

7. Farrow KN, Lee KJ, Perez M, et al. Brief hyperoxia increases mitochondrial oxidation and increases phosphodiesterase 5 activity in fetal pulmonary artery smooth muscle cells. Antioxid Redox Signal 2012;17:460-70.

8. O'Donovan DJ, Fernandes CJ. Free radicals and diseases in premature infants. Antioxid Redox Signal 2004;6:169-76.

9. Bhandari A, Bhandari V. Pitfalls, problems, and progress in bronchopulmonary dysplasia. Pediatrics 2009;123:1562-73.

10. Perlman JM, Wyllie J, Kattwinkel J, et al.; Neonatal Resuscitation Chapter Collaborators. Part 11: Neonatal resuscitation: 2010 International Consensus on Cardiopulmonary Resuscitation and Emergency Cardiovascular Care Science With Treatment Recommendations. Circulation 2010;122:16 Suppl 2:S516-38.

11. Vento M, Moro M, Escrig R, et al. Preterm resuscitation with low oxygen causes less oxidative stress, inflammation, and chronic lung disease. Pediatrics 2009;124:e439-49.

12. Munkeby BH, Børke WB, Bjørnland K, et al. Resuscitation of hypoxic piglets with $100 \%$ O2 increases pulmonary metalloproteinases and IL-8. Pediatr Res 2005;58:542-8.

13. Wagenaar GT, ter Horst SA, van Gastelen MA, et al. Gene expression profile and histopathology of experimental bronchopulmonary dysplasia induced by prolonged oxidative stress. Free Radic Biol Med 2004;36:782801.

14. Lee PJ, Choi AM. Pathways of cell signaling in hyperoxia. Free Radic Biol Med 2003;35:341-50.

15. Li Y, Arita Y, Koo HC, Davis JM, Kazzaz JA. Inhibition of c-Jun N-terminal kinase pathway improves cell viability in response to oxidant injury. Am J Respir Cell Mol Biol 2003;29:779-83.

16. Ben-Yosef Y, Lahat N, Shapiro S, Bitterman H, Miller A. Regulation of endothelial matrix metalloproteinase-2 by hypoxia/reoxygenation. Circ Res 2002;90:784-91.

17. Sejersted Y, Aasland AL, Bjørås M, Eide L, Saugstad OD. Accumulation of 8-oxoguanine in liver DNA during hyperoxic resuscitation of newborn mice. Pediatr Res 2009;66:533-8.

18. Truog WE, Xu D, Ekekezie II, et al. Chronic hypoxia and rat lung development: analysis by morphometry and directed microarray. Pediatr Res 2008;64:56-62.

19. Martín-Ancel A, García-Alix A, Gayá F, Cabañas F, Burgueros M, Quero J. Multiple organ involvement in perinatal asphyxia. J Pediatr 1995;127:78693.

20. Low JA. Intrapartum fetal asphyxia: definition, diagnosis, and classification. Am J Obstet Gynecol 1997;176:957-9.

21. Amy RW, Bowes D, Burri PH, Haines J, Thurlbeck WM. Postnatal growth of the mouse lung. J Anat 1977;124(Pt 1):131-51.

22. Heidecker B, Kasper EK, Wittstein IS, et al. Transcriptomic biomarkers for individual risk assessment in new-onset heart failure. Circulation 2008;118:238-46.

23. Gustavsson M, Wilson MA, Mallard C, Rousset C, Johnston MV, Hagberg H. Global gene expression in the developing rat brain after hypoxic preconditioning: involvement of apoptotic mechanisms? Pediatr Res 2007;61:444-50.
24. Janér J, Lassus P, Haglund C, Paavonen K, Alitalo K, Andersson S. Pulmonary vascular endothelial growth factor- $\mathrm{C}$ in development and lung injury in preterm infants. Am J Respir Crit Care Med 2006;174:326-30.

25. Remesal A, Pedraz C, San Feliciano L, Ludeña D. Pulmonary expression of vascular endothelial growth factor (VEGF) and alveolar septation in a newborn rat model exposed to acute hypoxia and recovered under conditions of air or hyperoxia. Histol Histopathol 2009;24:325-30.

26. O’Reilly MA, Staversky RJ, Watkins RH, Maniscalco WM, Keng PC. p53-independent induction of GADD45 and GADD153 in mouse lungs exposed to hyperoxia. Am J Physiol Lung Cell Mol Physiol 2000;278:L552-9.

27. Agorreta J, Zulueta JJ, Montuenga LM, Garayoa M. Adrenomedullin expression in a rat model of acute lung injury induced by hypoxia and LPS. Am J Physiol Lung Cell Mol Physiol 2005;288:L536-45.

28. Sciesielski LK, Paliege A, Martinka P, Scholz H. Enhanced pulmonary expression of the TrkB neurotrophin receptor in hypoxic rats is associated with increased acetylcholine-induced airway contractility. Acta Physiol (Oxf) 2009;197:253-64.

29. Abreu-Rodríguez I, Sánchez Silva R, Martins AP, et al. Functional and transcriptional induction of aquaporin-1 gene by hypoxia; analysis of promoter and role of Hif-1a. PLoS ONE 2011;6:e28385.

30. Cui LY, Yang S, Zhang J. Protective effects of neutrophil gelatinase-associated lipocalin on hypoxia/reoxygenation injury of HK-2 cells. Transplant Proc 2011;43:3622-7.

31. Roudkenar MH, Halabian R, Bahmani P, Roushandeh AM, Kuwahara Y, Fukumoto M. Neutrophil gelatinase-associated lipocalin: a new antioxidant that exerts its cytoprotective effect independent on Heme Oxygenase-1. Free Radic Res 2011;45:810-9.

32. Kumarasamy A, Schmitt I, Nave AH, et al. Lysyl oxidase activity is dysregulated during impaired alveolarization of mouse and human lungs. Am J Respir Crit Care Med 2009;180:1239-52.

33. Feng Z, Levine AJ. The regulation of energy metabolism and the IGF-1/ mTOR pathways by the p53 protein. Trends Cell Biol 2010;20:427-34.

34. Ahmad A, Ahmad S, Chang LY, Schaack J, White CW. Endothelial Akt activation by hyperoxia: role in cell survival. Free Radic Biol Med 2006;40:1108-18.

35. Carnesecchi S, Deffert C, Pagano A, et al. NADPH oxidase-1 plays a crucial role in hyperoxia-induced acute lung injury in mice. Am J Respir Crit Care Med 2009;180:972-81.

36. Houtgraaf JH, Versmissen J, van der Giessen WJ. A concise review of DNA damage checkpoints and repair in mammalian cells. Cardiovasc Revasc Med 2006;7:165-72.

37. Randerath E, Zhou GD, Randerath K. Organ-specific oxidative DNA damage associated with normal birth in rats. Carcinogenesis 1997;18:859-66.

38. Rancourt RC, Keng PC, Helt CE, O'Reilly MA. The role of p21(CIP1/ WAF1) in growth of epithelial cells exposed to hyperoxia. Am J Physiol Lung Cell Mol Physiol 2001;280:L617-26.

39. Dalen ML, Alme TN, Bjørås M, Munkeby BH, Rootwelt T, Saugstad OD. Reduced expression of DNA glycosylases in post-hypoxic newborn pigs undergoing therapeutic hypothermia. Brain Res 2010;1363:198-205.

40. Livak KJ, Schmittgen TD. Analysis of relative gene expression data using real-time quantitative PCR and the 2(-Delta Delta $C(T))$ Method. Methods 2001;25:402-8.

41. Irizarry RA, Hobbs B, Collin F, et al. Exploration, normalization, and summaries of high density oligonucleotide array probe level data. Biostatistics 2003;4:249-64.

42. Ishwaran H, Rao JS, Kogalur UB. BAMarraytrade mark: Java software for Bayesian analysis of variance for microarray data. BMC Bioinformatics 2006;7:59.

43. Subramanian A, Tamayo P, Mootha VK, et al. Gene set enrichment analysis: a knowledge-based approach for interpreting genome-wide expression profiles. Proc Natl Acad Sci USA 2005;102:15545-50.

44. Edgar R, Domrachev M, Lash AE. Gene Expression Omnibus: NCBI gene expression and hybridization array data repository. Nucleic Acids Res 2002;30:207-10. 